\title{
ON THE SIMPLICITY OF LIE ALGEBRAS ASSOCIATED TO LEAVITT ALGEBRAS
}

\author{
GENE ABRAMS AND DARREN FUNK-NEUBAUER
}

\begin{abstract}
For any field $\mathbb{K}$ and integer $n \geq 2$ we consider the Leavitt algebra $L=L_{\mathbb{K}}(n)$. $L$ is an associative algebra, but we view $L$ as a Lie algebra using the bracket $[a, b]=a b-b a$ for $a, b \in L$. We denote this Lie algebra as $L^{-}$, and consider its Lie subalgebra $\left[L^{-}, L^{-}\right]$. In our main result, we show that $\left[L^{-}, L^{-}\right]$is a simple Lie algebra if and only if char $(\mathbb{K})$ divides $n-1$. For any positive integer $d$ we let $S=M_{d}\left(L_{\mathbb{K}}(n)\right)$ be the $d \times d$ matrix algebra over $L_{\mathbb{K}}(n)$. We give sufficient conditions for the simplicity and non-simplicity of the Lie algebra $\left[S^{-}, S^{-}\right]$.
\end{abstract}

\section{INTRODUCTION}

In the seminal paper [9], Leavitt constructed and investigated properties of associative rings $R$ which lack the invariant basis property; that is, rings for which two free left $R$ modules of different finite rank are isomorphic. In particular, for each integer $n \geq 2$ and each field $\mathbb{K}$, Leavitt constructed the (now-so-called) Leavitt $\mathbb{K}$-algebra of type $(1, n)$, denoted in this paper by $L_{\mathbb{K}}(n)$. The algebra $R=L_{\mathbb{K}}(n)$ has the property that ${ }_{R} R \cong{ }_{R} R^{n}$ as left $R$-modules.

The study of Leavitt algebras has enjoyed a recent resurgence, as these algebras have come to be viewed as specific (important) cases of the so-called Leavitt path algebras (see e.g. [2]). Furthermore, the Leavitt algebras were re-discovered in [11], where they arise as quotients of the deep matrix algebras. In [8] the study of Lie algebras associated to deep matrix algebras and these quotients is taken up.

The purpose of this paper is threefold. First, we provide a detailed description of the relationship between the deep matrix algebras of finite type (as described in [11] and [7]) and the Leavitt algebras. Second, we prove our main result: the Lie algebra $\left[L_{\mathbb{K}}(n)^{-}, L_{\mathbb{K}}(n)^{-}\right]$ is simple if and only if $\operatorname{char}(\mathbb{K})$ divides $n-1$. Finally, we discuss the $d \times d$ matrix algebra over $L_{\mathbb{K}}(n)$, denoted $M_{d}\left(L_{\mathbb{K}}(n)\right)$, and prove three propositions which extend to $M_{d}\left(L_{\mathbb{K}}(n)\right)$ the ideas underlying our main result.

Throughout the paper we let $\mathbb{K}$ denote any field and $1_{\mathbb{K}}$ denote the multiplicative identity of $\mathbb{K}$. We begin by formally defining the algebras of interest.

Definition 1.1. Let $n \geq 2$ be an integer, and $\mathbb{K}$ be any field.

(i) The Cohn $\mathbb{K}$-algebra of order $n$, denoted $C_{\mathbb{K}}(n)$, is the unital associative $\mathbb{K}$-algebra with generators $x_{i}, y_{i}(1 \leq i \leq n)$ and relations $y_{i} x_{j}=\delta_{i, j} 1_{C(n)}(1 \leq i, j \leq n)$ where

2000 Mathematics Subject Classification. Primary 16D30, 16S99, $17 \mathrm{~B} 65$.

Key words and phrases. Leavitt algebra, Leavitt path algebra, simple Lie algebra.

The first author is partially supported by the U.S. National Security Agency under grant number H8923009-1-0066. 
$1_{C(n)}$ denotes the multiplicative identity of $C_{\mathbb{K}}(n)$.

(ii) Let $M$ denote the two sided ideal of $C_{\mathbb{K}}(n)$ generated by the element $1_{C(n)}-\sum_{i=1}^{n} x_{i} y_{i}$. The Leavitt $\mathbb{K}$-algebra of order $n$, denoted $L_{\mathbb{K}}(n)$, is the quotient algebra $C_{\mathbb{K}}(n) / M$.

For convenience we will often denote $C_{\mathbb{K}}(n)\left(\operatorname{resp} . L_{\mathbb{K}}(n)\right)$ by $C(n)$ (resp. $\left.L(n)\right)$. Additionally, we let $-: C_{\mathbb{K}}(n) \rightarrow L_{\mathbb{K}}(n)$ denote the canonical quotient map, so that, for $z \in C_{\mathbb{K}}(n)$, $z+M \in L_{\mathbb{K}}(n)$ will often be denoted by $\bar{z}$.

We note that $L_{\mathbb{K}}(n)$ may also be defined as the $\mathbb{K}$-algebra generated by the same generators and relations as those of $C_{\mathbb{K}}(n)$, plus the additional relation $1_{C(n)}=\sum_{i=1}^{n} x_{i} y_{i}$. We choose to present the above quotient definition of $L_{\mathbb{K}}(n)$ to make some of our results more transparent. In particular, note that $1_{L(n)}=\overline{1_{C(n)}}=1_{C(n)}+M$.

Although the Cohn and Leavitt algebras are associative algebras, our main interest in this paper is with Lie algebras. Any associative $\mathbb{K}$-algebra $A$ can be turned into a Lie algebra over $\mathbb{K}$ in the following way: for $a, b \in A$ we define the Lie bracket $[a, b]=a b-b a$ (where $a b$, $b a$ denote the associative product in $A$ ). We denote this Lie algebra structure on $A$ by $A^{-}$.

We recall here a few basic definitions concerning Lie algebras (for more information see [6]). Let $\mathfrak{L}$ denote a Lie algebra over $\mathbb{K}$. [ $[\mathfrak{L}, \mathfrak{L}]$ denotes the Lie subalgebra of $\mathfrak{L}$ consisting of all finite sums of elements of the form $[a, b]$ where $a, b \in \mathfrak{L}$. A subset $\mathfrak{I}$ of $\mathfrak{L}$ is an $i d e a l$ of $\mathfrak{L}$ if $\mathfrak{I}$ is a $\mathbb{K}$-subspace of the vector space $\mathfrak{L}$ and $[\mathfrak{L}, \mathfrak{I}] \subseteq \mathfrak{I}$. The Lie algebra $\mathfrak{L}$ is called simple if $[\mathfrak{L}, \mathfrak{L}] \neq\{0\}$, and the only ideals of $\mathfrak{L}$ are $\{0\}$ and $\mathfrak{L}$.

To provide some context for our main result we present the following theorem from classical Lie theory. For an integer $d \geq 2$ let $M_{d}(\mathbb{K})$ denote the $d \times d$ matrix algebra over $\mathbb{K}$. Then $M_{d}(\mathbb{K})^{-}=\mathfrak{g l}_{d}(\mathbb{K})$, the general linear Lie algebra over $\mathbb{K}$. Let $\mathfrak{s l}_{d}(\mathbb{K})$ denote the Lie subalgebra of $\mathfrak{g l}_{d}(\mathbb{K})$ consisting of trace zero matrices. It is well known that $\left[\mathfrak{g l}_{d}(\mathbb{K}), \mathfrak{g l}_{d}(\mathbb{K})\right]=\mathfrak{s l}_{d}(\mathbb{K})$ (see [6, p. 9]) and that $\mathfrak{s l}_{d}(\mathbb{K})$ is simple if and only if char $(\mathbb{K})$ does not divide $d$ (see [12, Chap. 2]). From these comments we obtain the following theorem.

Theorem. For any field $\mathbb{K}$ and integer $d \geq 2$, the Lie algebra $\left[M_{d}(\mathbb{K})^{-}, M_{d}(\mathbb{K})^{-}\right]$is simple if and only if char $(\mathbb{K})$ does not divide $d$.

We now state our main result, which we will prove in Section 4 .

Theorem 1.2. For any field $\mathbb{K}$ and integer $n \geq 2$, the Lie algebra $\left[L_{\mathbb{K}}(n)^{-}, L_{\mathbb{K}}(n)^{-}\right]$is simple if and only if char $(\mathbb{K})$ divides $n-1$.

We note that the above theorem corrects [8, Theorem 6.10], and extends the result to all fields (including those of characteristic 2). (In [8] the Lie algebra $\left[L_{\mathbb{K}}(n)^{-}, L_{\mathbb{K}}(n)^{-}\right]$is denoted by $\mathfrak{s l a}$.) We also note that both of the Lie algebras $L_{\mathbb{K}}(n)^{-}$and $\left[L_{\mathbb{K}}(n)^{-}, L_{\mathbb{K}}(n)^{-}\right]$ are infinite dimensional $\mathbb{K}$-vector spaces (see Lemma 4.2).

\section{Preliminaries}

In this section we present some notation and results concerning the Cohn algebra which will be used throughout the paper. 
Throughout the rest of the paper $n$ will denote an integer greater than or equal to 2 , and $X$ will denote the set $\{1,2, \ldots, n\}$.

Definition 2.1. Let $\operatorname{Mon}(X)$ denote the free monoid generated by $X$. That is, the elements of $\operatorname{Mon}(X)$ are finite words in the alphabet $X$ and multiplication is given by concatenation. The identity element of $\operatorname{Mon}(X)$ is the empty word, denoted $\phi$. For nonempty $I \in \operatorname{Mon}(X)$ we write $I=i_{1} i_{2} \ldots i_{t}$ with $t \in \mathbb{Z}^{+}$and $i_{s} \in X(1 \leq s \leq t)$ and refer to $t$ as the length of $I$; we define the length of $\phi$ to be 0 . We define $I^{\text {rev }}=i_{t} i_{t-1} \ldots i_{1}$ and $\phi^{\text {rev }}=\phi$.

Note 2.2. The following facts follow immediately from Definition 2.1, For all $I, J \in M o n(X)$ we have $\left(I^{r e v}\right)^{r e v}=I$ and $(I J)^{r e v}=J^{r e v} I^{r e v}$.

Definition 2.3. $\operatorname{Mon}(X)$ is equipped with the following partial order.

(i) For $I, J \in \operatorname{Mon}(X)$ we define $I \leq J$ to mean there exists $K \in \operatorname{Mon}(X)$ such that $J=I K$.

(ii) For $I, J \in M o n(X)$ we write $I \sim J$ if either $I \leq J$ or $J \leq I$. We write $I \nsim J$ if $I \not J J$ and $J \not \leq I$

Notation 2.4. For a nonempty $I \in \operatorname{Mon}(X)$ with $I=i_{1} i_{2} \ldots i_{t}$ we let $x_{I}$ (resp. $y_{I}$ ) abbreviate the element $x_{i_{1}} x_{i_{2}} \ldots x_{i_{t}}$ (resp. $\left.y_{i_{1}} y_{i_{2}} \ldots y_{i_{t}}\right)$ of $C(n)$. We let $x_{\phi}$ and $y_{\phi}$ both abbreviate the element $1_{C(n)}$ of $C(n)$.

Note 2.5. The following facts follow immediately from Definition 1.1 and Notation 2.4. For all $I, J \in \operatorname{Mon}(X)$ we have $x_{I J}=x_{I} x_{J}$ and $y_{I J}=y_{I} y_{J}$. Also, for all $I \in \operatorname{Mon}(X)$ we have $y_{I} x_{I^{\text {rev }}}=1_{C(n)}$ and $y_{I^{\text {rev }}} x_{I}=1_{C(n)}$.

Next we present two lemmas: one which gives a basis for the Cohn algebra $C(n)$ and one which describes how to multiply two elements in this basis.

Lemma 2.6. The set $\left\{x_{I} y_{J} \mid I, J \in M o n(X)\right\}$ is a basis for $C(n)$.

Proof: Immediate from Definition 1.1(i).

Lemma 2.7. For all $I, J, K, L \in M o n(X)$ the product of basis elements in $C(n)$ is given by $\left(x_{I} y_{J}\right)\left(x_{K} y_{L}\right)=\left\{\begin{array}{cl}0 & \text { if } J^{\text {rev }} \nsim K, \\ x_{I M} y_{L} & \text { if } J^{\text {rev }} \leq K \text { with } K=J^{\text {rev }} M \text { for some } M \in M \text { on }(X), \\ x_{I} y_{N^{r e v} L} & \text { if } K \leq J^{\text {rev }} \text { with } J^{\text {rev }}=K N \text { for some } N \in M \text { on }(X) .\end{array}\right.$

Proof: Case 1: Suppose $J^{r e v} \nsim K$. Then the product $\left(x_{I} y_{J}\right)\left(x_{K} y_{L}\right)=0$ since by Definition 1.1(i) we have $y_{i} x_{j}=0$ for $i \neq j$.

Case 2: Suppose $J^{r e v} \leq K$ with $K=J^{r e v} M$ for some $M \in M o n(X)$. From this and Note 2.5 we have $\left(x_{I} y_{J}\right)\left(x_{K} y_{L}\right)=x_{I} y_{J} x_{J^{r e v}} x_{M} y_{L}=x_{I M} y_{L}$.

Case 3: Suppose $K \leq J^{r e v}$ with $J^{r e v}=K N$ for some $N \in M o n(X)$. Observe by Note 2.2 that $J=N^{r e v} K^{r e v}$. From this and Note 2.5 we have $\left(x_{I} y_{J}\right)\left(x_{K} y_{L}\right)=x_{I} y_{N^{r e v}} y_{K^{r e v}} x_{K} y_{L}=$ $x_{I} y_{N^{r e v} L}$.

We now briefly discuss the deep matrix algebras of finite type and their connection to the algebras in this paper. See [8] for further details. Recall for an integer $n \geq 2$ that $X$ 
denotes the set $\{1,2, \ldots, n\}$. The deep matrix algebra $\mathcal{D} \mathcal{M}(X, \mathbb{K})$ is the unital associative $\mathbb{K}$-algebra with basis elements $\mathfrak{e}(h, k)$ indexed by pairs $h, k \in \operatorname{Mon}(X)$. The multiplication of these basis elements is defined in [8] in a fashion similar to the multiplication from Lemma 2.7. The deep matrix algebra $\mathcal{D} \mathcal{M}(X, \mathbb{K})$ is isomorphic to the Cohn algebra $C_{\mathbb{K}}(n)$. In particular, a straightforward check yields that the following map $\psi: \mathcal{D} \mathcal{M}(X, \mathbb{K}) \rightarrow C_{\mathbb{K}}(n)$ is an isomorphism of $\mathbb{K}$-algebras: let $\psi$ be the unique linear transformation determined by the basis assignments $\psi(\mathfrak{e}(I, J))=x_{I} y_{J^{r e v}}$. Furthermore, in [8] the author defines $\mathcal{M}$ to be the two sided ideal of $\mathcal{D} \mathcal{M}(X, \mathbb{K})$ generated by the element $\mathfrak{e}(\phi, \phi)-\sum_{x \in X} \mathfrak{e}(x, x)$. Observe that $\psi(\mathcal{M})=M$ (where $M$ is the ideal of $C_{\mathbb{K}}(n)$ presented in Definition 1.1(ii)) and so $\mathcal{D} \mathcal{M}(X, \mathbb{K}) / \mathcal{M}$ and $L_{\mathbb{K}}(n)$ are isomorphic as $\mathbb{K}$-algebras.

\section{The trace Functions $T$ And $\tau$}

In this section we introduce certain linear transformations $T: C_{\mathbb{K}}(n) \rightarrow \mathbb{K}$ and $\tau: L_{\mathbb{K}}(n) \rightarrow \mathbb{K}$ which will be used to prove that if $\operatorname{char}(\mathbb{K})$ divides $n-1$ then the Lie algebra $\left[L_{\mathbb{K}}(n)^{-}, L_{\mathbb{K}}(n)^{-}\right]$is simple (see Theorem 1.2$)$. These transformations are referred to as trace functions because they satisfy properties similar to those of the usual trace function on matrices.

Recall that, by Lemma 2.6, the set $\left\{x_{I} y_{J} \mid I, J \in \operatorname{Mon}(X)\right\}$ is a basis for $C_{\mathbb{K}}(n)$.

Definition 3.1. Let $T: C_{\mathbb{K}}(n) \rightarrow \mathbb{K}$ denote the unique $\mathbb{K}$-linear transformation determined by the basis assignments:

$$
T\left(x_{I} y_{J}\right)=\left\{\begin{array}{cl}
0 & \text { if } I \neq J^{r e v} \\
1_{\mathbb{K}} & \text { if } I=J^{r e v}
\end{array}\right.
$$

In particular, $T\left(1_{C(n)}\right)=T\left(x_{\phi} y_{\phi}\right)=1_{\mathbb{K}}$.

We now establish some properties of the linear transformation $T$.

Lemma 3.2. For all $A, B, C \in M o n(X)$ and $i \in X$ we have

(i) $T\left(x_{A B} y_{C A^{\text {rev }}}\right)=T\left(x_{B} y_{C}\right)$.

(ii) $T\left(x_{A^{\text {rev }} B} y_{C A}\right)=T\left(x_{B} y_{C}\right)$.

(iii) $T\left(x_{A i} y_{i B}\right)=T\left(x_{A} y_{B}\right)$.

Proof: (i): By Note 2.2 we have $\left(C A^{r e v}\right)^{r e v}=A C^{\text {rev }}$. Using this we find that $B=C^{r e v}$ if and only if $A B=\left(C A^{r e v}\right)^{r e v}$. Using this and Definition 3.1 we obtain the result.

(ii): Immediate from (i) and the fact that $\left(A^{r e v}\right)^{r e v}=A$.

(iii): By Note $2.2(i B)^{r e v}=B^{r e v} i^{r e v}=B^{r e v} i$. From this we have that $A=B^{r e v}$ if and only if $A i=(i B)^{r e v}$. Using this and Definition 3.1 we obtain the result.

The next result establishes that $T: C_{\mathbb{K}}(n) \rightarrow \mathbb{K}$ satisfies a property analogous to a property of the standard trace function on matrix algebras over $\mathbb{K}$.

Proposition 3.3. For all $c, c^{\prime} \in C(n)$ we have $T\left(c c^{\prime}\right)=T\left(c^{\prime} c\right)$.

Proof: By Lemma 2.6 and since $T$ is linear it suffices to show that for all $I, J, K, L \in \operatorname{Mon}(X)$

$$
T\left(\left(x_{I} y_{J}\right)\left(x_{K} y_{L}\right)\right)=T\left(\left(x_{K} y_{L}\right)\left(x_{I} y_{J}\right)\right) \text {. }
$$


Referring to Definition 2.3 we break the proof into four cases depending on whether or not $J^{r e v} \sim K$ and whether or not $L^{r e v} \sim I$.

Case 1: Suppose $J^{r e v} \nsim K$ and $L^{r e v} \nsim I$. By Lemma 2.7 it is immediate that (1) holds.

Case 2: Suppose $J^{r e v} \nsim K$ and $L^{\text {rev }} \sim I$. Then either $L^{r e v} \leq I$ or $I \leq L^{r e v}$. We show that (1) holds in the case $L^{r e v} \leq I$. The case $I \leq L^{r e v}$ is proved similarly. By Lemma 2.7

$$
\left(x_{I} y_{J}\right)\left(x_{K} y_{L}\right)=0 \text {. }
$$

Since $L^{r e v} \leq I$ there exists an $I^{\prime} \in \operatorname{Mon}(X)$ such that $I=L^{r e v} I^{\prime}$. From this and Note 2.5 we have $\left(x_{K} y_{L}\right)\left(x_{I} y_{J}\right)=x_{K} y_{L} x_{L^{r e v}} x_{I^{\prime}} y_{J}=x_{K I^{\prime}} y_{J}$. Since $J^{r e v} \nsim K$ then $K I^{\prime} \neq J^{r e v}$. Combining the previous two sentences we have

$$
T\left(\left(x_{K} y_{L}\right)\left(x_{I} y_{J}\right)\right)=0 .
$$

Combining (2) and (3) we obtain (11).

Case 3: Suppose $J^{r e v} \sim K$ and $L^{r e v} \nsim I$. Similar to Case 2.

Case 4: Suppose $J^{r e v} \sim K$ and $L^{r e v} \sim I$. We break this case into four subcases depending on whether $J^{r e v} \leq K$ or $K \leq J^{r e v}$ and whether $L^{r e v} \leq I$ or $I \leq L^{r e v}$.

Subcase (i): Suppose $J^{r e v} \leq K$ and $I \leq L^{r e v}$. Then there exists $K^{\prime}, L^{\prime} \in \operatorname{Mon}(X)$ such that $K=J^{r e v} K^{\prime}$ and $L^{r e v}=I L^{\prime}$. By Note $2.2 L=\left(L^{\prime}\right)^{r e v} I^{r e v}$ and so using Note 2.5 we have $\left(x_{I} y_{J}\right)\left(x_{K} y_{L}\right)=x_{I} y_{J} x_{J^{r e v}} x_{K^{\prime}} y_{\left(L^{\prime}\right)^{r e v}} y_{I^{r e v}}=x_{I K^{\prime}} y_{\left(L^{\prime}\right)^{r e v} I^{r e v}}$. From this and Lemma 3.2(i) we have

$$
T\left(\left(x_{I} y_{J}\right)\left(x_{K} y_{L}\right)\right)=T\left(x_{K^{\prime}} y_{\left(L^{\prime}\right)^{r e v}}\right) .
$$

Similarly we have $\left(x_{K} y_{L}\right)\left(x_{I} y_{J}\right)=x_{J^{r e v}} x_{K^{\prime}} y_{\left(L^{\prime}\right)^{\text {rev }}} y_{I^{\text {rev }}} x_{I} y_{J}=x_{J^{r e v} K^{\prime}} y_{\left(L^{\prime}\right)^{r e v} J}$. From this and Lemma 3.2 (ii) we have

$$
T\left(\left(x_{K} y_{L}\right)\left(x_{I} y_{J}\right)\right)=T\left(x_{K^{\prime}} y_{\left(L^{\prime}\right)^{r e v}}\right) .
$$

Combining (44) and (5) we obtain (1).

Subcase (ii): Suppose $K \leq J^{r e v}$ and $L^{r e v} \leq I$. Similar to Subcase (i).

Subcase (iii): Suppose $J^{r e v} \leq K$ and $L^{r e v} \leq I$. Then there exists $K^{\prime}, I^{\prime} \in M o n(X)$ such that $K=J^{r e v} K^{\prime}$ and $I=L^{r e v} I^{\prime}$. By Note2.5 we have $\left(x_{I} y_{J}\right)\left(x_{K} y_{L}\right)=x_{L^{\text {rev }}} x_{I^{\prime}} y_{J} x_{J^{r e v}} x_{K^{\prime}} y_{L}$ $=x_{L^{r e v} I^{\prime} K^{\prime}} y_{\phi L}$. From this and Lemma 3.2 (ii) we have

$$
T\left(\left(x_{I} y_{J}\right)\left(x_{K} y_{L}\right)\right)=T\left(x_{I^{\prime} K^{\prime}} y_{\phi}\right)=\left\{\begin{array}{cl}
0 & \text { if } K^{\prime} \neq \phi \text { or } I^{\prime} \neq \phi \\
1_{\mathbb{K}} & \text { if } K^{\prime}=\phi \text { and } I^{\prime}=\phi .
\end{array}\right.
$$

Similarly we have $\left(x_{K} y_{L}\right)\left(x_{I} y_{J}\right)=x_{J^{\text {rev }}} x_{K^{\prime}} y_{L} x_{L^{\text {rev }}} x_{I^{\prime}} y_{J}=x_{J^{\text {rev }} K^{\prime} I^{\prime}} y_{\phi J}$. From this and Lemma 3.2 (ii) we have

$$
T\left(\left(x_{K} y_{L}\right)\left(x_{I} y_{J}\right)\right)=T\left(x_{K^{\prime} I^{\prime}} y_{\phi}\right)=\left\{\begin{array}{cl}
0 & \text { if } K^{\prime} \neq \phi \text { or } I^{\prime} \neq \phi \\
1_{\mathbb{K}} & \text { if } K^{\prime}=\phi \text { and } I^{\prime}=\phi .
\end{array}\right.
$$

Combining (6) and (7) we obtain (1). 
Subcase (iv): Suppose $K \leq J^{r e v}$ and $I \leq L^{\text {rev }}$. Similar to Subcase (iii).

We now establish an important property of $T$, and subsequently use it to define the linear transformation $\tau: L_{\mathbb{K}}(n) \rightarrow \mathbb{K}$. While each of the two previous results were independent of the field $\mathbb{K}$, the next result imposes a relationship between char $(\mathbb{K})$ and $n$.

Lemma 3.4. Assume char $(\mathbb{K})$ divides $n-1$. Then $T(m)=0$ for all $m \in M$.

Proof: By Definition 1.1(ii) and Lemma 2.6 we have

$$
M=\operatorname{span}_{\mathbb{K}}\left\{x_{I} y_{J}\left(1_{C(n)}-\sum_{i=1}^{n} x_{i} y_{i}\right) x_{K} y_{L} \mid I, J, K, L \in M o n(X)\right\} .
$$

Thus it suffices to show that for all $I, J, K, L \in \operatorname{Mon}(X)$

$$
T\left(x_{I} y_{J}\left(1_{C(n)}-\sum_{i=1}^{n} x_{i} y_{i}\right) x_{K} y_{L}\right)=0 .
$$

We break the proof into three cases depending on whether $J \neq \phi, K \neq \phi$, or $J=K=\phi$.

Case 1: Suppose $J \neq \phi$. Using Note 2.2 and Lemma 2.7 we have for $1 \leq i \leq n$

$$
x_{I} y_{J} x_{i} y_{i}=\left\{\begin{array}{cl}
x_{I} y_{J} & \text { if } i \text { is the first letter of the word } J^{\text {rev }} \\
0 & \text { otherwise. }
\end{array}\right.
$$

From this we have $x_{I} y_{J}\left(1_{C(n)}-\sum_{i=1}^{n} x_{i} y_{i}\right) x_{K} y_{L}=\left(x_{I} y_{J}-\sum_{i=1}^{n} x_{I} x_{J} x_{i} y_{i}\right) x_{K} y_{L}=0$ and so we obtain (8).

Case 2: Suppose $K \neq \phi$. Similar to Case 1 .

Case 3: Suppose $J=K=\phi$. Recall $x_{\phi}=y_{\phi}=1_{C(n)}$ and observe that $x_{I} y_{\phi}\left(1_{C(n)}-\sum_{i=1}^{n} x_{i} y_{i}\right) x_{\phi} y_{L}=x_{I} y_{L}-\sum_{i=1}^{n} x_{I i} y_{i L}$. From this and Lemma 3.2(iii) we have $T\left(x_{I} y_{\phi}\left(1_{C(n)}-\sum_{i=1}^{n} x_{i} y_{i}\right) x_{\phi} y_{L}\right)=T\left(x_{I} y_{L}\right)-\sum_{i=1}^{n} T\left(x_{I} y_{L}\right)=\left\{\begin{array}{cl}0 & \text { if } I \neq L^{r e v} \\ 1_{\mathbb{K}}-n 1_{\mathbb{K}} & \text { if } I=L^{r e v}\end{array}\right.$,

Since $\operatorname{char}(\mathbb{K})$ divides $n-1$ we have $1_{\mathbb{K}}-n 1_{\mathbb{K}}=-(n-1) 1_{\mathbb{K}}=0$ in $\mathbb{K}$. Combining the previous two sentences we obtain (8).

Recall by Definition 1.1(ii) that $L_{\mathbb{K}}(n)$ is defined as the quotient $C_{\mathbb{K}}(n) / M$.

Definition 3.5. Assume char $(\mathbb{K})$ divides $n-1$. We define

$$
\tau: L_{\mathbb{K}}(n) \rightarrow \mathbb{K}
$$

as the $\mathbb{K}$-linear transformation given by $\tau(c+M)=T(c)$ for $c \in C_{\mathbb{K}}(n)$. We note that an application of Lemma 3.4 is required to ensure that $\tau$ is well defined.

Note 3.6. By Definition 3.5 we have $\tau\left(1_{L(n)}\right)=\tau\left(1_{C(n)}+M\right)=T\left(1_{C(n)}\right)=T\left(x_{\phi} y_{\phi}\right)=1_{\mathbb{K}}$.

Proposition 3.7. Assume char $(\mathbb{K})$ divides $n-1$. Then the linear transformation $\tau: L_{\mathbb{K}}(n) \rightarrow \mathbb{K}$ has the following properties:

(i) $\tau\left(l l^{\prime}\right)=\tau\left(l^{\prime} l\right)$ for all $l, l^{\prime} \in L_{\mathbb{K}}(n)$.

(ii) $\tau\left(1_{L(n)}\right) \neq 0$ in $\mathbb{K}$. 
Proof: (i): Immediate from Proposition 3.3 and Definition 3.5 .

(ii): Immediate from Note 3.6.

\section{The MAIn RESUlT}

In this section we prove Theorem 1.2, We begin by presenting a number of results which will be used in the proof. As a reminder, we denote by $-: C_{\mathbb{K}}(n) \rightarrow L_{\mathbb{K}}(n)$ the canonical quotient map.

Lemma 4.1. Let $\left\{I_{v} \mid 1 \leq v \leq V\right\}$ be a set of distinct elements of Mon $(X)$, for some positive integer $V$. Then the set $\left\{\overline{x_{I_{v}}} \mid 1 \leq v \leq V\right\}$ is linearly independent in $L_{\mathbb{K}}(n)$.

Proof: This follows directly from [13, Lemma 1.1], a result which applies more generally to all Leavitt path algebras. For clarity and completeness, we present here a proof which applies to the context at hand.

By a $\mathbb{Z}$-grading on an associative $\mathbb{K}$-algebra $A$ we mean a direct sum decomposition $A=\oplus_{n \in \mathbb{Z}} A_{n}$ as $\mathbb{K}$-vector spaces, for which $A_{n} A_{m} \subseteq A_{n+m}$ for all $n, m \in \mathbb{Z}$. $A_{n}$ is called the component of degree $n$. There is a standard $\mathbb{Z}$-grading on the free associative $\mathbb{K}$-algebra $F$ in the $2 n$ variables $\left\{x_{1}, \ldots, x_{n}, y_{1}, \ldots, y_{n}\right\}$, generated by defining $\operatorname{deg}\left(x_{i}\right)=1$ and $\operatorname{deg}\left(y_{i}\right)=-1$ for $1 \leq i \leq n$. (So, for instance, $x_{1} x_{2} y_{2} x_{1} \in F_{2}$.) We may view $L_{\mathbb{K}}(n)$ as the quotient algebra of $F$ by the two sided ideal generated by the element $1_{C(n)}-\sum_{i=1}^{n} x_{i} y_{i}$, together with the elements $y_{i} x_{j}-\delta_{i, j} 1_{C(n)}(1 \leq i, j \leq n)$. Since each of these generating elements lies in $F_{0}$, the quotient algebra $L_{\mathbb{K}}(n)$ naturally inherits the $\mathbb{Z}$-grading from $F$.

We now prove the result of interest. We note that each element of $L_{\mathbb{K}}(n)$ of the form $\overline{x_{I}}$ has degree greater than or equal to 0 , since the degree of each individual $\overline{x_{i}}$ is 1 . Suppose we have

$$
\sum_{v=1}^{V} k_{v} \overline{x_{I_{v}}}=0
$$

in $L_{\mathbb{K}}(n)$, where $k_{v} \in \mathbb{K}(1 \leq v \leq V)$. To obtain the result we show $k_{v}=0(1 \leq v \leq V)$. Since the $\mathbb{Z}$-grading on $L_{\mathbb{K}}(n)$ produces by definition a direct sum decomposition, we can assume without loss of generality that for each non-negative integer $m$,

$$
\sum k_{w} \overline{x_{I_{w}}}=0
$$

where the sum is taken over all $I_{w}$ for which the length of $I_{w}$ is $m$.

Now fix $m \geq 0$. For each $s(1 \leq s \leq V)$ such that the length of $I_{s}$ equals $m$, multiply the displayed expression (9) on the left by $\overline{y_{s}^{\text {rev }}}$. Since all of the indices $I_{w}$ in (9) have length $m$ then by Definition 1.1 the expression $\overline{y_{I_{s}^{r e v}}} \cdot \overline{x_{I_{w}}}$ is either 0 (if and only if $I_{s} \neq I_{w}$ ), or $1_{L(n)}$ (if and only if $I_{s}=I_{w}$ ). In this way we conclude that $k_{w}=0$ for each $k_{w}$ in the sum given in (9). Since this same conclusion can be drawn for each nonnegative integer $m$, we have $k_{v}=0$ for all $1 \leq v \leq V$.

Lemma 4.2. Let $\mathbb{K}$ be any field, and $n \geq 2$.

(i) $\left[L_{\mathbb{K}}(n)^{-}, L_{\mathbb{K}}(n)^{-}\right]$is infinite dimensional over $\mathbb{K}$.

(ii) $L_{\mathbb{K}}(n)^{-}$is infinite dimensional over $\mathbb{K}$. 
(iii) Let $W$ denote $\left[L_{\mathbb{K}}(n)^{-}, L_{\mathbb{K}}(n)^{-}\right]$. Then $[W, W] \neq\{0\}$.

Proof: (i): Using Lemma 4.1 and the definition of $[-,-]$, we see that the set $\left\{\left[\overline{x_{1}}, \overline{x_{2}}{ }^{j}\right] \mid j \in \mathbb{Z}, j \geq 1\right\}$ is $\mathbb{K}$-linearly independent, and the result follows.

(ii): Immediate from (i) and that $\left[L_{\mathbb{K}}(n)^{-}, L_{\mathbb{K}}(n)^{-}\right] \subseteq L_{\mathbb{K}}(n)^{-}$.

(iii): Again using Lemma 4.1 and the definition of $[-,-]$, the element $\left.\left[\overline{x_{1}}, \overline{x_{2}}\right],\left[\overline{x_{1}}, \overline{x_{2}}{ }^{2}\right]\right]$ of $[W, W]$ is nonzero, and the result follows.

Let $A$ denote an associative $\mathbb{K}$-algebra. Let $Z(A)$ denote the center of $A$, that is, $Z(A)=\left\{a \in A \mid a a^{\prime}=a^{\prime} a\right.$ for all $\left.\mathrm{a}^{\prime} \in \mathrm{A}\right\}$.

We will utilize the following three Theorems to achieve our main result. The first of these Theorems is due to Herstein.

Theorem. ([5, Theorem 1.13]) Let $A$ denote a simple associative ring. Assume it is not the case that $\operatorname{char}(A)=2$ and $A$ is 4-dimensional over $Z(A)$. Then $U \subseteq Z(A)$ for any proper Lie ideal $U$ of the Lie algebra $\left[A^{-}, A^{-}\right]$.

We will apply [5, Theorem 1.13] to the associative ring $L(n)$. Therefore we will need the following theorem, which was established by Leavitt in the early 1960's, and rediscovered recently by Faulkner - McCrimmon and Kennedy.

Theorem. ([10, Theorem 2]) For any field $\mathbb{K}$ and integer $n \geq 2, L_{\mathbb{K}}(n)$ is a simple ring.

In [4, Theorem 4.1] and [7, Theorem 3] the above theorem is proven in the context of deep matrix algebras. We note that the the simplicity of $L_{\mathbb{K}}(n)$ does not depend on the field $\mathbb{K}$.

When applying [5, Theorem 1.13] to $L(n)$ the following theorem will be useful.

Theorem. ([3, Theorem 4.2] or [7, Theorem 15]) The center of $L_{\mathbb{K}}(n)$ is one dimensional; that is,

$$
Z\left(L_{\mathbb{K}}(n)\right)=\mathbb{K} 1_{L(n)}=\left\{\alpha 1_{L(n)} \mid \alpha \in \mathbb{K}\right\} .
$$

We combine the previous three theorems to get the following result, which will be the main ingredient in the proof of Theorem 1.2.

Proposition 4.3. Let $\mathbb{K}$ be any field, and $n \geq 2$. Then $1_{L(n)} \notin\left[L(n)^{-}, L(n)^{-}\right]$if and only if the Lie algebra $\left[L(n)^{-}, L(n)^{-}\right]$is simple.

Proof: Let $W$ denote the Lie algebra $\left[L(n)^{-}, L(n)^{-}\right]$.

$(\Longleftarrow)$ : Assume $1_{L(n)} \in W$. We prove $W$ is not simple. We have $\mathbb{K} 1_{L(n)} \subseteq W$. Clearly, $\mathbb{K} 1_{L(n)} \neq\{0\}$ and by Lemma 4.2(i) $\mathbb{K} 1_{L(n)} \neq W$. Also, $\mathbb{K} 1_{L(n)}$ is a $\mathbb{K}$-subspace of $W$ and $\left[W, \mathbb{K} 1_{L(n)}\right]=\{0\} \subseteq \mathbb{K} 1_{L(n)}$. Thus, $\mathbb{K} 1_{L(n)}$ is a nonzero, proper Lie ideal of $W$ and so $W$ is not simple.

$(\Longrightarrow)$ : Assume $1_{L(n)} \notin W$. We prove $W$ is simple. By Lemma 4.2(iii) we have $[W, W] \neq\{0\}$. Thus it suffices to show that the only ideals of $W$ are $\{0\}$ and $W$. Let $U$ denote a proper 
Lie ideal of $W$. We show $U=\{0\}$. By [10, Theorem 2] $L(n)$ is a simple ring. From Lemma 4.2(ii) we find $L(n)$ is not 4-dimensional over $Z(L(n))$. Thus, by [5, Theorem 1.13] and [3, Theorem 4.2] we find $U \subseteq \mathbb{K} 1_{L(n)}$. Therefore, since $1_{L(n)} \notin W$ we have $U \subseteq \mathbb{K} 1_{L(n)} \cap W=\{0\}$ and so $U=\{0\}$.

We are now ready to prove our main result.

Proof of Theorem 1.2: $\quad(\Longleftarrow)$ : Assume char $(\mathbb{K})$ divides $n-1$. We prove the Lie algebra $\left[L(n)^{-}, L(n)^{-}\right]$is simple. By Proposition 4.3 it suffices to show that $1_{L(n)} \notin\left[L(n)^{-}, L(n)^{-}\right]$. By contradiction, suppose $1_{L(n)} \in\left[L(n)^{-}, L(n)^{-}\right]$; so there exist a positive integer $r$, and $l_{i}, l_{i}^{\prime} \in L(n)^{-}(1 \leq i \leq r)$ such that

$$
1_{L(n)}=\sum_{i=1}^{r}\left[l_{i}, l_{i}^{\prime}\right] .
$$

By Proposition 3.7(ii), $\tau\left(1_{L(n)}\right) \neq 0$ in $\mathbb{K}$. On the other hand, using Proposition 3.7(i), we have $\tau\left(\sum_{i=1}^{r}\left[l_{i}, l_{i}^{\prime}\right]\right)=\sum_{i=1}^{r} \tau\left(l_{i} l_{i}^{\prime}\right)-\tau\left(l_{i}^{\prime} l_{i}\right)=0$, thereby yielding the desired contradiction.

$(\Longrightarrow)$ : Assume char $(\mathbb{K})$ does not divide $n-1$. We prove the Lie algebra $\left[L(n)^{-}, L(n)^{-}\right]$is not simple. By Proposition 4.3 it suffices to show $1_{L(n)} \in\left[L(n)^{-}, L(n)^{-}\right]$. Let $-: C(n) \rightarrow L(n)$ denote the canonical quotient map, that is, $\bar{c}=c+M$ for all $c \in C(n)$. By Definition 1.1(ii) we have

$$
\overline{1_{C(n)}}=\sum_{i=1}^{n} \overline{x_{i}} \overline{y_{i}}
$$

and so

$$
\begin{aligned}
\sum_{i=1}^{n}\left[\overline{y_{i}}, \overline{x_{i}}\right] & =\sum_{i=1}^{n}\left(\overline{y_{i} x_{i}}-\overline{x_{i}} \overline{y_{i}}\right) \\
& =\sum_{i=1}^{n} \overline{1_{C(n)}}-\sum_{i=1}^{n} \overline{x_{i}} \overline{y_{i}} \\
& =n \overline{1_{C(n)}}-\overline{1_{C(n)}} \\
& =(n-1) 1_{L(n)} .
\end{aligned}
$$

Using the hypothesis that $\operatorname{char}(\mathbb{K})$ does not divide $n-1$, we multiply both sides of the above equation by $(n-1)^{-1}$ and conclude that $1_{L(n)} \in\left[L(n)^{-}, L(n)^{-}\right]$.

\section{An EXTENSION TO MATRIX ALgEBRAS OVER $L_{\mathbb{K}}(n)$}

The collection of matrix algebras of the form $S=M_{d}\left(L_{\mathbb{K}}(n)\right)$ for positive integers $d$ has been the subject of a number of investigations (see e.g. [1]). In this section we give sufficient conditions for both the simplicity and non-simplicity of the Lie algebras $\left[S^{-}, S^{-}\right]$in terms of the relationship between the three integers $\operatorname{char}(\mathbb{K}), n$, and $d$. Our sufficient conditions are effective enough to allow us to obtain Theorem 1.2 as a consequence. However, we are currently unable to obtain a set of necessary and sufficient conditions for the simplicity of 
$\left[S^{-}, S^{-}\right]$in all cases.

We start with a general ring-theoretic result.

Proposition 5.1. Suppose $R$ is a unital ring with the property that ${ }_{R} R \cong{ }_{R} R^{m}$ as left $R$-modules for some integer $m>1$. Then $(m-1) 1_{R} \in\left[R^{-}, R^{-}\right]$.

Proof: As observed in [9], an isomorphism ${ }_{R} R \cong{ }_{R} R^{m}$ yields the existence of $2 m$ elements $x_{1}, \ldots, x_{m}, y_{1}, \ldots, y_{m}$ in $R$ such that $y_{i} x_{j}=\delta_{i, j} 1_{R}$, and $\sum_{i=1}^{m} x_{i} y_{i}=1_{R}$. (This follows directly from the realization of a homomorphism having domain a free left module of rank 1 as right multiplication by an element in the range module.) But then

$$
\sum_{i=1}^{m}\left[y_{i}, x_{i}\right]=\sum_{i=1}^{m}\left(1_{R}-x_{i} y_{i}\right)=m 1_{R}-\sum_{i=1}^{m} x_{i} y_{i}=m 1_{R}-1_{R}=(m-1) 1_{R} .
$$

We establish our results about algebras of the form $M_{d}\left(L_{\mathbb{K}}(n)\right)$ by invoking the following generalization of Proposition 4.3.

Proposition 5.2. Let $\mathbb{K}$ be any field, and $n$, d positive integers with $n \geq 2$. Let $S$ denote $M_{d}\left(L_{\mathbb{K}}(n)\right)$. Then $1_{S} \notin\left[S^{-}, S^{-}\right]$if and only if the Lie algebra $\left[S^{-}, S^{-}\right]$is simple.

Proof: Each of the three Theorems presented immediately prior to Proposition 4.3 hold for $M_{d}\left(L_{\mathbb{K}}(n)\right)$. (Note in particular that the simplicity of $M_{d}\left(L_{\mathbb{K}}(n)\right)$ and the one-dimensionality of the center of $M_{d}\left(L_{\mathbb{K}}(n)\right)$ follow from the corresponding properties of $L_{\mathbb{K}}(n)$ and standard ring theory). So the proof given of Proposition 4.3 generalizes immediately to the matrix rings $M_{d}\left(L_{\mathbb{K}}(n)\right)$ as well.

We let

$$
g=g . c . d \cdot(d, n-1) .
$$

Proposition 5.3. Let $\mathbb{K}$ be any field and let $n, d$ be positive integers with $n \geq 2$. Let $S$ denote $M_{d}\left(L_{\mathbb{K}}(n)\right)$. If char $(\mathbb{K})$ does not divide $\frac{n-1}{g}$, then the Lie algebra $\left[S^{-}, S^{-}\right]$is not simple.

Proof: In [9, Theorem 5], Leavitt established that there is an isomorphism ${ }_{S} S \cong{ }_{S} S^{\frac{n-1}{g}+1}$ of left $S$-modules. So applying Proposition [5.1, we have $\left(\left(\frac{n-1}{g}+1\right)-1\right) 1_{S}=\left(\frac{n-1}{g}\right) 1_{S} \in\left[S^{-}, S^{-}\right]$. Now the hypothesis gives that $\frac{n-1}{g} \neq 0$ in $\mathbb{K}$, so that by dividing we get $1_{S} \in\left[S^{-}, S^{-}\right]$. The result now follows from Proposition 5.2 .

Lemma 5.4. Suppose $A$ is an associative $\mathbb{K}$-algebra admitting a trace function; that is, suppose there exists a $\mathbb{K}$-linear functional tr $: A \rightarrow \mathbb{K}$ for which $\operatorname{tr}\left(a a^{\prime}\right)=\operatorname{tr}\left(a^{\prime} a\right)$ for all $a, a^{\prime} \in A$. Let $d$ be any positive integer. Then the matrix algebra $M_{d}(A)$ admits a trace function $\operatorname{tr}_{d}$ by setting

$$
\operatorname{tr}_{d}(B)=\sum_{i=1}^{d} \operatorname{tr}\left(B_{i, i}\right)
$$

for each $B \in M_{d}(A)$. 
Proof: The proof that $t r_{d}$ is a $\mathbb{K}$-linear functional is routine. The fact that $t r_{d}(B C)=$ $\operatorname{tr}_{d}(C B)$ for all $B, C \in M_{d}(A)$ follows directly from the definition of matrix multiplication and the fact that $\operatorname{tr}\left(a a^{\prime}\right)=\operatorname{tr}\left(a^{\prime} a\right)$ for all $a, a^{\prime} \in A$.

Now we have a partial converse of Proposition 5.3 .

Proposition 5.5. Let $\mathbb{K}$ be any field and let $n, d$ be positive integers with $n \geq 2$. Let $S$ denote $M_{d}\left(L_{\mathbb{K}}(n)\right)$. If char $(\mathbb{K})$ divides $\frac{n-1}{g}$ and char $(\mathbb{K})$ does not divide $d$, then the Lie algebra $\left[S^{-}, S^{-}\right]$is simple.

Proof: By Proposition 5.2 it suffices to show that $1_{S} \notin\left[S^{-}, S^{-}\right]$. Since char $(\mathbb{K})$ divides $\frac{n-1}{g}$ we have in particular that $\operatorname{char}(\mathbb{K})$ divides $n-1$. Thus the trace function $\tau: L_{\mathbb{K}}(n) \rightarrow \mathbb{K}$ as given in Definition 3.5 is well defined. By Proposition 3.7(i) and Lemma 5.4 there exists a trace function $\tau_{d}: S \rightarrow \mathbb{K}$. In particular, since $\tau_{d}(B C)=\tau_{d}(C B)$ for any two elements $B, C \in S$ we have

$$
\tau_{d}(x)=0 \text { for any } x \in\left[S^{-}, S^{-}\right] .
$$

Now by the definition of $\tau_{d}$ and Note 3.6 we have $\tau_{d}\left(1_{S}\right)=\sum_{i=1}^{d} \tau\left(1_{L(n)}\right)=d \cdot \tau\left(1_{L(n)}\right)=d 1_{\mathbb{K}}$. Since $\operatorname{char}(\mathbb{K})$ does not divide $d$ we get that $d 1_{\mathbb{K}}=\tau_{d}\left(1_{S}\right)$ is nonzero in $\mathbb{K}$, which by (10) gives $1_{S} \notin\left[S^{-}, S^{-}\right]$.

When $d=1$ then $S=M_{d}\left(L_{\mathbb{K}}(n)\right)$ is isomorphic to $L_{\mathbb{K}}(n)$. Furthermore, the case $d=1$ yields the hypothesis that char $(\mathbb{K})$ does not divide $n-1$ in Proposition 5.3 , and the hypothesis that $\operatorname{char}(\mathbb{K})$ does divide $n-1$ in Proposition 5.5. Thus these two Propositions together yield Theorem 1.2 .

It would be interesting to find necessary and sufficient conditions for the simplicity of $\left[S^{-}, S^{-}\right]$. More generally, suppose $E$ is a finite directed graph, and let $R=L_{\mathbb{K}}(E)$ denote the Leavitt path algebra of $E$ with coefficients in $\mathbb{K}$ (see e.g. [2]). The Leavitt path algebras include as specific examples not only the algebras $L_{\mathbb{K}}(n)$, but the matrix $\operatorname{rings} M_{d}(\mathbb{K})$ and $M_{d}\left(L_{\mathbb{K}}(n)\right)$ as well. It would be interesting to find necessary and sufficient conditions on $\mathbb{K}$ and $E$ so that the Lie algebra $\left[L_{\mathbb{K}}(E)^{-}, L_{\mathbb{K}}(E)^{-}\right]$is simple.

\section{REFERENCES}

[1] G. Abrams, P.N. Ánh, E. Pardo, Isomorphisms between Leavitt algebras and their matrix rings, J. reine angew. Math. 624 (2008), 103-132.

[2] G. Abrams, G. Aranda Pino, The Leavitt path algebra of a graph, J. Algebra 293 (2005), 319-334.

[3] G. Aranda Pino, K. Crow The Center of a Leavitt path algebra, preprint.

[4] J. Faulkner, K. MCCrimmon Finitely deep matrices, Comm. Alg. 36 (2008), 3897-3911.

[5] I.N. Herstein, "Topics in Ring Theory", University of Chicago Mathematics Lecture Notes, 1965.

[6] J. Humphreys, "Introduction to Lie algebras and representation theory", Springer-Verlag Inc., 1972.

[7] C. Kennedy, Deep matrix algebras of finite type, Algebr. Represent.Theor. 9 (2006), 525-537.

[8] C. Kennedy, Simple and nearly simple deep matrix algebras, Algebr. Represent. Theor. (to appear), DOI 10.1007/s10468-008-9127-0.

[9] W.G. Leavitt, The module type of a ring, Trans. A.M.S. 103 (1962), 113-130.

[10] W.G. Leavitt, The module type of homomorphic images, Duke Math. J. 32 (1965), 305-311.

[11] K. MCCRimmon, Deep matrices and their Frankenstein actions, in: "Non-Associative Algebra and its Applications (Sabinen, Sbitneva, Shestakov, eds.)", Lecture notes in Pure and Applied Mathematics 246, CRC Publishers, Boca Raton (2006). 
[12] G.B. Seligman, "Modular Lie algebras", Springer-Verlag Inc., 1967.

[13] M. Siles Molina, Algebras of quotients of path algebras, J. Algebra 319 (2008), 5265-5278.

Department of Mathematics, University of Colorado, Colorado Springs CO 80933 U.S.A.

E-mail address: abrams@math.uccs.edu

Department of Mathematics, Colorado State University, Pueblo CO 81001 U.S.A.

E-mail address: darren.funkneubauer@colostate-pueblo.edu 